PROCEEDINGS OF THE

AMERICAN MATHEMATICAL SOCIETY

Volume 134, Number 2, Pages 411-418

S 0002-9939(05)07982-7

Article electronically published on August 25, 2005

\title{
FIXED POINT THEOREMS IN ORDERED $L$-SPACES
}

\author{
ADRIAN PETRUŞEL AND IOAN A. RUS
}

(Communicated by Joseph A. Ball)

\begin{abstract}
The purpose of this paper is to present some fixed point results in ordered L-spaces. Our results generalize and extend a recent result of Ran and Reurings (2004). Some applications to matrix equations are also considered.
\end{abstract}

\section{INTRODUCTION}

Recently, in a paper of Ran-Reurings (see [9]), the following result was proved:

Theorem 1.1 (Ran-Reurings 9]). Let $X$ be a partially ordered set such that every pair $x, y \in X$ has a lower and an upper bound. Let $d$ be a metric on $X$ such that the metric space $(X, d)$ is complete. Let $f: X \rightarrow X$ be a continuous and monotone (i.e., either order-preserving or order-reversing) operator. Suppose that the following two assertions hold:

1) there exists $a \in] 0,1[$ such that $d(f(x), f(y)) \leq a \cdot d(x, y)$, for each $x, y \in X$ with $x \geq y$,

2) there exists $x_{0} \in X$ such that $x_{0} \leq f\left(x_{0}\right)$ or $x_{0} \geq f\left(x_{0}\right)$.

Then $f$ has a unique fixed point $x^{*} \in X$, i.e., $f\left(x^{*}\right)=x^{*}$, and for each $x \in X$ the sequence $\left(f^{n}(x)\right)_{n \in \mathbb{N}}$ of successive approximations of $f$ starting from $x$ converges to $x^{*} \in X$.

Usually, when a set is endowed with a metric and an order structure, an additional assumption is added, namely the compatibility between the above structures, i.e.,

$$
\left(x_{n}\right)_{n \in \mathbb{N}} \rightarrow x,\left(y_{n}\right)_{n \in \mathbb{N}} \rightarrow y \text { and } x_{n} \leq y_{n}, \text { for each } n \in \mathbb{N} \Rightarrow x \leq y .
$$

Following the proof of Ran and Reurings result, we may remark that the conclusion follows from the relations

$d\left(f^{n}\left(x_{1}\right), f^{n}(x)\right) \leq a^{n} \cdot d\left(x_{1}, x\right)$ and $d\left(f^{n}(x), f^{n}\left(x_{2}\right)\right) \leq a^{n} \cdot d\left(x, x_{2}\right), x_{1} \leq x \leq x_{2}$,

by letting $n \rightarrow+\infty$.

Dropping the hypothesis that each pair of points has an upper and a lower bound, we immediately obtain the following result.

Theorem 1.2. Let $X$ be a partially ordered set and let $d$ be a metric on $X$ such that the metric space $(X, d)$ is complete and the metric and the ordered structure

Received by the editors June 18, 2004 and, in revised form, September 1, 2004

2000 Mathematics Subject Classification. Primary 47H10; Secondary 54H25, 15A24.

(C)2005 American Mathematical Society 
are compatible. Let $f: X \rightarrow X$ be a continuous and monotone (i.e., either orderpreserving or order-reversing) operator. Suppose that the following two assertions hold:

1) there exists $a \in] 0,1[$ such that $d(f(x), f(y)) \leq a \cdot d(x, y)$, for each $x, y \in X$ with $x \geq y$,

2) there exists $x_{0} \in X$ such that $x_{0} \leq f\left(x_{0}\right)$ or $x_{0} \geq f\left(x_{0}\right)$.

Then $f$ has at least a fixed point $x^{*} \in X$ and for each $x \in X$ with $x \geq x_{0}$ (or $x \leq x_{0}$ ) the sequence $\left(f^{n}(x)\right)_{n \in \mathbb{N}}$ of successive approximations of $f$ starting from $x$ converges to $x^{*} \in X$.

The purpose of this paper is to generalize and extend Theorem 1.1 and Theorem 1.2 for the case of an ordered L-space.

\section{Notations AND BASiC NOTIONS}

Let $f: X \rightarrow X$ be an operator. Then $f^{0}:=1_{X}, f^{1}:=f, \ldots, f^{n+1}=f \circ f^{n}, n \in$ $\mathbb{N}$, denote the iterate operators of $f$. By $I(f)$ we will denote the set of all nonempty invariant subsets of $f$, i.e., $I(f):=\{Y \subset X \mid f(Y) \subseteq Y\}$.

Also, by $F_{f}:=\{x \in X \mid x=f(x)\}$ we will denote the fixed point set of the operator $f$, while $A_{f}\left(x^{*}\right):=\left\{x \in X \mid f^{n}(x) \rightarrow x^{*}\right.$, as $\left.n \rightarrow+\infty\right\}$ denotes the attractor basin of $f$ with respect to $x^{*} \in X$.

Let $X$ be a nonempty set. Denote $s(X):=\left\{\left(x_{n}\right)_{n \in N} \mid x_{n} \in X, n \in N\right\}$.

Let $c(X) \subset s(X)$ be a subset of $s(X)$ and let Lim $: c(X) \rightarrow X$ be an operator. By definition, the triple $(X, c(X), \operatorname{Lim})$ is called an L-space (Fréchet [3]) if the following conditions are satisfied:

(i) If $x_{n}=x, \forall n \in N$, then $\left(x_{n}\right)_{n \in N} \in c(X)$ and $\operatorname{Lim}\left(x_{n}\right)_{n \in N}=x$.

(ii) If $\left(x_{n}\right)_{n \in N} \in c(X)$ and $\operatorname{Lim}\left(x_{n}\right)_{n \in N}=x$, then for all subsequences, $\left(x_{n_{i}}\right)_{i \in N}$ of $\left(x_{n}\right)_{n \in N}$ we have that $\left(x_{n_{i}}\right)_{i \in N} \in c(X)$ and $\operatorname{Lim}\left(x_{n_{i}}\right)_{i \in N}=x$.

By definition an element of $c(X)$ is a convergent sequence, $x:=\operatorname{Lim}\left(x_{n}\right)_{n \in N}$ is the limit of this sequence and we also write $x_{n} \rightarrow x$ as $n \rightarrow+\infty$.

In what follows we denote an L-space by $(X, \rightarrow)$.

In this setting, an operator $f: X \rightarrow X$ is called orbitally continuous if $x \in X$ and $f^{n(i)}(x) \rightarrow a \in X$, as $i \rightarrow+\infty$ imply $f^{n(i)+1}(x) \rightarrow f(a)$, as $i \rightarrow+\infty$.

Let $(X, \leq)$ be an partially ordered set. Denote $X_{\leq}:=\{(x, y) \in X \times X \mid x \leq$ $y$ or $y \leq x\}$. Also, if $x, y \in X$, with $x \leq y$, then by $[x, y]_{\leq}$we will denote the ordered segment joining $x$ and $y$, i.e., $[x, y]_{\leq}:=\{z \in X \mid x \leq z \leq y\}$. In the same setting, consider $f: X \rightarrow X$. Then, $(L F)_{f}:=\{x \in X \mid x \leq f(x)\}$ is the lower fixed point set of $f$, while $(U F)_{f}:=\{x \in X \mid x \geq f(x)\}$ is the upper fixed point set of $f$. Also, if $f: X \rightarrow X$ and $g: Y \rightarrow Y$, then the cartezian product of $f$ and $g$ is denoted by $f \times g$, and it is defined in the following way: $f \times g: X \times Y \rightarrow$ $X \times Y,(f \times g)(x, y):=(f(x), g(y))$.

Definition 2.1. Let $X$ be a nonempty set. Then, by definition, $(X, \rightarrow, \leq)$ is an ordered L-space if and only if:

(i) $(X, \rightarrow)$ is an L-space,

(ii) $(X, \leq)$ is a partially ordered set,

(iii) $\left(x_{n}\right)_{n \in \mathbb{N}} \rightarrow x,\left(y_{n}\right)_{n \in \mathbb{N}} \rightarrow y$ and $x_{n} \leq y_{n}$, for each $n \in \mathbb{N} \Rightarrow x \leq y$.

Throughout this paper we suppose that $(X, \rightarrow, \leq)$ is an ordered L-space satisfying the following additional assertion:

(iv) $\left(x_{n}\right)_{n \in \mathbb{N}} \rightarrow x,\left(z_{n}\right)_{n \in \mathbb{N}} \rightarrow x$ and $x_{n} \leq y_{n} \leq z_{n}$, for each $n \in \mathbb{N} \Rightarrow y_{n} \rightarrow x$. 
For more considerations on convergence structures see P. P. Zabreiko [13] and De Pascale, Marino and Pietromala [2].

\section{Picard ANd Weakly PiCARd operators}

First recall the following important concept.

Definition 3.1 (I.A. Rus [1]). Let $(X, \rightarrow)$ be an L-space. An operator $f: X \rightarrow X$ is, by definition, a Picard operator (briefly PO) if:

(i) $F_{f}=\left\{x^{*}\right\}$,

(ii) $\left(f^{n}(x)\right)_{n \in \mathbb{N}} \rightarrow x^{*}$ as $n \rightarrow \infty$, for all $x \in X$.

In terms of the Picard operators, some classical results in metrical fixed point theory have the following form $([6],[10])$.

Example 3.2 (Contraction principle). Let $(X, d)$ be a complete metric space and let $f: X \rightarrow X$ be an $a$-contraction, i.e., $a \in] 0,1[$ and $d(f(x), f(y)) \leq a \cdot d(x, y)$, for each $x, y \in X$. Then $f$ is a $\mathrm{PO}$.

Example 3.3 (Nemytzki and Edelstein). Let $(X, d)$ be a compact metric space and let $f: X \rightarrow X$ satisfy $d(f(x), f(y))<d(x, y)$, for all $x, y \in X$ with $x \neq y$. Then $f$ is a $\mathrm{PO}$.

Example 3.4 (Perov). Let $(X, d)$ be a complete generalized metric space $\left(d(x, y) \in \mathbb{R}_{+}^{m}\right)$ and $A \in M_{m m}\left(\mathbb{R}_{+}\right)$, such that, $A^{n} \rightarrow 0$ as $n \rightarrow \infty$. If $f: X \rightarrow X$ is an $A$-contraction, i.e., $d(f(x), f(y)) \leq A d(x, y)$, for all $x, y \in X$, then $f$ is a PO.

Example 3.5 (Sehgal and Bharucha-Reid, [5]). Let $(X, F, \min )$ be a complete probabilistic metric space. Let $f: X \rightarrow X$ be a continuous operator for which there exists $a \in\left[0,1\left[\operatorname{such}\right.\right.$ that $F_{f(x), f(y)}(a \lambda) \geq F_{x, y}(\lambda)$, for each $x, y \in X$ and each $\lambda>0$. Then $f$ is a PO.

Another important concept is:

Definition 3.6. Let $(X, \rightarrow)$ be an L-space. By definition, $f: X \rightarrow X$ is called a weakly Picard operator (briefly WPO) if the sequence $\left(f^{n}(x)\right)_{n \in N}$ converges for all $x \in X$ and the limit (which may depend on $x$ ) is a fixed point of $f$.

Example 3.7. Let $(X, d)$ be a complete metric space and let $f: X \rightarrow X$ be an orbitally continuous operator such that there is $a \in] 0,1[$ with the property $d\left(f(x), f^{2}(x)\right) \leq a \cdot d(x, f(x))$, for each $x \in X$. Then $f$ is a WPO.

Example 3.8. Let $(X, d)$ be a complete metric space, let $f: X \rightarrow X$ be an orbitally continuous operator and let $\varphi: X \rightarrow \mathbb{R}_{+}$. We suppose that $f$ satisfies the Caristi condition with respect to $\varphi$, i.e., $d(x, f(x)) \leq \varphi(x)-\varphi(f(x))$, for each $x \in X$. Then $f$ is a WPO.

In I. A. Rus [11 the basic theory of Picard and weakly Picard operators is presented. For the multivalued case, see Petruşel [8]. For both settings, see also [12. 


\section{FiXed POINT RESUlts}

Our starting result is the following abstract lemma.

Lemma 4.1. Let $(X, \rightarrow)$ be an L-space and let $U$ be a symmetric subset of $X \times X$ such that $\Delta(X)$ (the diagonal of $X \times X$ ) is included in $U$. Let $f: X \rightarrow X$ be an operator. Suppose that:

(i) for each $x, y \in X$ there exists $z \in X$ such that $(x, z) \in U$ and $(y, z) \in U$,

(ii) there exist $x_{0}, x^{*} \in X$ such that $x_{0} \in A_{f}\left(x^{*}\right)$,

(iii) $(x, y) \in U$ and $x \in A_{f}\left(x^{*}\right)$ implies $y \in A_{f}\left(x^{*}\right)$.

Then $A_{f}\left(x^{*}\right)=X$. Moreover, if in addition the operator $f$ is orbitally continuous, then $F_{f}=\left\{x^{*}\right\}$.

Proof. Let $x \in X$ be arbitrary. If $\left(x_{0}, x\right) \in U$, then the conclusion follows from (ii) and (iii). Suppose $\left(x_{0}, x\right) \notin U$. Then from (i) there exists $z \in X$ such that $\left(x_{0}, z\right) \in U$ and $(x, z) \in U$. From (ii) and (iii) we obtain that $x \in A_{f}\left(x^{*}\right)$. The second part of the theorem follows from the orbital continuity of $f$. $U$.

Some consequences of the above abstract result follow by particular choices of

Theorem 4.2. Let $(X, \rightarrow, \leq)$ be an ordered L-space and let $f: X \rightarrow X$ be an operator. We suppose that:

(i) For each $x, y \in X$ there exist $m(x, y), M(x, y) \in X$ such that $x, y \in[m(x, y)$, $M(x, y)]_{\leq}$.

(ii) $f:(X, \rightarrow) \rightarrow(X, \rightarrow)$ is orbitally continuous.

(iii) There exist $x_{0}, x^{*} \in X$ such that $\left(f^{n}\left(x_{0}\right)\right)_{n \in \mathbb{N}} \rightarrow x^{*}$, as $n \rightarrow+\infty$.

(iv) If $(x, y) \in X_{\leq}$and $\left(f^{n}(x)\right)_{n \in \mathbb{N}} \rightarrow x^{*}$, as $n \rightarrow+\infty$, then $\left(f^{n}(y)\right)_{n \in \mathbb{N}} \rightarrow x^{*}$, as $n \rightarrow+\infty$.

Then $f$ is a $P O$.

Proof. A. A direct proof. From (ii) and (iii) we have that $x^{*} \in F_{f}$. Let $x \in X$ be arbitrary. If $\left(x, x_{0}\right) \in X_{\leq}$, then the conclusion follows from (iii) and (iv). If $\left(x, x_{0}\right) \notin X_{\leq}$, then by (i) we have that $x, x_{0} \in\left[m\left(x, x_{0}\right), M\left(x, x_{0}\right)\right]_{\leq}$. Since $x_{0} \in\left[m\left(x, x_{0}\right), M\left(x, x_{0}\right)\right]_{\leq}$and taking into account of (iv), it follows that

$$
\left(f^{n}\left(m\left(x, x_{0}\right)\right)\right)_{n \in \mathbb{N}} \rightarrow x^{*} \text { and }\left(f^{n}\left(M\left(x, x_{0}\right)\right)\right)_{n \in \mathbb{N}} \rightarrow x^{*} \text {, as } n \rightarrow+\infty \text {. }
$$

From the fact that $m\left(x, x_{0}\right) \leq x \leq M\left(x, x_{0}\right)$ and (iv) we obtain $\left(f^{n}(x)\right)_{n \in \mathbb{N}} \rightarrow x^{*}$, as $n \rightarrow+\infty$. Hence, $f$ is a $\mathrm{PO}$ with respect to $\rightarrow$.

B. A proof based on Lemma 4.1. Take $U:=X_{\leq}$and apply Lemma 4.1.

From Theorem 4.2. we have the following result.

Theorem 4.3. Let $(X, d, \leq)$ be an ordered metric space and let $f: X \rightarrow X$ be an operator. We suppose that:

(a) for each $x, y \in X$ there exist $m(x, y), M(x, y) \in X$ such that $x, y \in[m(x, y)$, $M(x, y)]_{\leq}$

(b) $X_{\leq} \in I(f \times f)$.

(c) $f:(X, d) \rightarrow(X, d)$ is orbitally continuous.

(d) The metric $d$ is complete.

(e) There exists $x_{0} \in X$ such that $\left(x_{0}, f\left(x_{0}\right)\right) \in X_{\leq}$.

(f) There exists $\alpha \in] 0,1\left[\right.$ such that $d(f(x), f(y)) \leq \alpha \cdot d(x, y)$, for each $x, y \in X_{\leq}$.

Then $f:(X, d) \rightarrow(X, d)$ is a $P O$. 
Proof. Let $x_{0} \in X$ such that $\left(x_{0}, f\left(x_{0}\right)\right) \in X_{\leq}$. From (b), (d) and (f) it follows that there exists $x^{*} \in X$ such that, with respect to the metric $d$, we have $\left(f^{n}\left(x_{0}\right)\right)_{n \in \mathbb{N}} \rightarrow$ $x^{*}$, as $n \rightarrow+\infty$. From (c) we get that $x^{*} \in F_{f}$.

If $\left(x, x_{0}\right) \in X_{\leq}$, then, from (c), (f) and (a) we have that $\left(f^{n}(x)\right)_{n \in \mathbb{N}} \rightarrow x^{*}$ (with respect to $d)$, as $n \rightarrow+\infty$.

If $\left(x, x_{0}\right) \notin X_{<}$, then, from (f), (c) and (b), we get the same conclusion.

Our result follows now from Theorem 4.2., namely the case when the L-structure of the space is that induced by the metric $d$.

Remark 4.4. The conclusion of Theorem 4.3 holds if instead the hypothesis (b) we put:

$\left(\mathrm{b}^{\prime}\right) f:(X, \leq) \rightarrow(X, \leq)$ is monotone increasing

or

$\left(\mathrm{b}^{\prime \prime}\right) f:(X, \leq) \rightarrow(X, \leq)$ is monotone decreasing.

Of course, it is easy to remark that assertion (b) in Theorem 4.3 is more general. For example, if we consider the ordered $L$-space $\left(\mathbb{R}^{2}, \rightarrow, \leq\right)$, then $f: \mathbb{R}^{2} \rightarrow$ $\mathbb{R}^{2}, f(x, y):=(\varphi(x, y) \cdot \varphi(x, y))$ satisfies (ii), for all $\varphi: \mathbb{R}^{2} \rightarrow \mathbb{R}$.

Remark 4.5. Condition (e) from the above theorem is equivalent to:

$\left(\mathrm{e}^{\prime}\right)(L F)_{f} \cup(U F)_{f} \neq \emptyset$.

Remark 4.6. Theorem 1.1 (see Ran and Reurings [9]) is a particular case of Theorem 4.2 and Theorem 4.3 .

Similarly we can establish the following results.

Theorem 4.7. Let $(X, d, \leq)$ be an ordered generalized metric space (in the sense that $d(x, y) \in \mathbb{R}_{+}^{n}$ ) and let $f: X \rightarrow X$ be an operator. We suppose that:

(a) For each $x, y \in X$ there exist $m(x, y), M(x, y) \in X$ such that $x, y \in[m(x, y)$, $M(x, y)]_{\leq}$.

(b) $X_{\leq} \in I(f \times f)$.

(c) $f:(X, d) \rightarrow(X, d)$ is orbitally continuous.

(d) The metric $d$ is complete.

(e) There exists $x_{0} \in X$ such that $\left(x_{0}, f\left(x_{0}\right)\right) \in X_{\leq}$.

(f) There exists a matrix $A \in M_{m m}\left(\mathbb{R}_{+}\right)$with $A^{n} \rightarrow 0$ as $n \rightarrow \infty$, such that $d(f(x), f(y)) \leq A \cdot d(x, y)$, for each $x, y \in X_{\leq}$.

Then $f:(X, d) \rightarrow(X, d)$ is a PO.

Theorem 4.8. Let $(X, F$, min, $\leq)$ be an ordered probabilistic complete metric space and let $f: X \rightarrow X$ be an operator. We suppose that:

(a) For each $x, y \in X$ there exist $m(x, y), M(x, y) \in X$ such that $x, y \in[m(x, y)$, $M(x, y)]_{\leq}$.

(b) $X_{\leq} \in I(f \times f)$.

(c) $f:(X, F, \min ) \rightarrow(X, F$, min $)$ is orbitally continuous.

(d) $(L F)_{f} \cup(U F)_{f} \neq \emptyset$.

(e) There exists $a \in\left[0,1\left[\right.\right.$ such that $F_{f(x), f(y)}(a \lambda) \geq F_{x, y}(\lambda)$, for each $x, y \in X_{\leq}$ and each $\lambda>0$.

Then $f:(X, d) \rightarrow(X, d)$ is a PO.

Proof. From (d) there exists $x_{0} \in(L F)_{f} \cup(U F)_{f}$. Then from (b) and (e) we get that $\left(f^{n}\left(x_{0}\right)\right)_{n \in \mathbb{N}} \rightarrow x^{*}$, as $n \rightarrow+\infty$, for some $x^{*} \in X$. From (c) we get that $x^{*} \in F_{f}$. 
Further on, if $\left(x, x_{0}\right) \in X_{\leq}$, then, from (b) and (e) we have that $\left(f^{n}(x)\right)_{n \in \mathbb{N}} \rightarrow$ $x^{*}$, as $n \rightarrow+\infty$.

If $\left(x, x_{0}\right) \notin X_{\leq}$, then, from (a), (b) and (e) and we get the same conclusion.

Our result now follows from Theorem 4.2. namely the particular case when the L-structure of the space is that induced by the probabilistic metric one.

Remark 4.9. Theorem 4.7 is an extension of Perov's fixed point theorem, while Theorem 4.8 generalizes Sehgal and Bharucha-Reid's fixed point principle. It is worth noting that other fixed point results can be established by replacing the contraction condition on $f$ with a generalized contraction assertion (see [6], 1], [10], etc.).

Also, it is not difficult to see that more general fixed point results in probabilistic metric spaces can be proved in this manner. For example, we also have the following generalization of Theorem 4.8 (see Hadžić and Pap [5] for the notions involved).

Theorem 4.10. Let $(X, F, T, \leq)$ be an ordered complete Menger space, let $T$ be a t-norm of H-type and let $f: X \rightarrow X$ be an operator. We suppose that:

(a) For each $x, y \in X$ there exist $m(x, y), M(x, y) \in X$ such that $x, y \in[m(x, y)$, $M(x, y)]_{\leq}$.

(b) $X_{\leq} \in I(f \times f)$.

(c) $f:(X, F, T) \rightarrow(X, F, T)$ is orbitally continuous.

(d) $(L F)_{f} \cup(U F)_{f} \neq \emptyset$.

(e) There exists $a \in\left[0,1\left[\right.\right.$ such that $F_{f(x), f(y)}(a \lambda) \geq F_{x, y}(\lambda)$, for each $x, y \in X_{\leq}$ and each $\lambda>0$.

Then $f:(X, d) \rightarrow(X, d)$ is a $P O$.

\section{Applications}

Consider first the equation

$$
X=Q+\sum_{j=1}^{m} A_{j}^{*} \cdot g(X) \cdot A_{j},
$$

where $Q$ is a positive definite matrix, $A_{j}$ are arbitrary $n \times n$ matrices and the map $g: \mathcal{P}(n) \rightarrow \mathcal{P}(n)$. Denote by $\mathcal{H}(n)$ the set of all Hermitian matrices and by $\mathcal{P}(n) \subset \mathcal{H}(n)$ the set of all $n \times n$ positive definite matrices. $X \in \mathcal{P}(n)$ will be briefly denoted $X>0$. See $[9$ for more details.

The next theorem is an extension of Theorem 4.1 and Theorem 4.2 in RanReurings [9], not only with respect to the continuity, but mainly with respect to the monotonicity condition on $g$.

Theorem 5.1. Let $Q \in \mathcal{P}(n)$ and let $g: \mathcal{P}(n) \rightarrow \mathcal{P}(n)$ be orbitally continuous. Suppose that:

(a) there exists $M>0$ such that:

(i) $\sum_{j=1}^{m} A_{j}^{*} A_{j}<M \cdot I_{n}$,

(ii) $\|\operatorname{tr}(g(Y)-g(X))\| \leq M^{-1} \cdot\|\operatorname{tr}(Y-X)\|$, for each $X \leq Y$.

(b) $\mathcal{H}(n)_{\leq} \in I(g \times g)$.

Then equation (5.1) has a unique solution $X^{*}$ in $\mathcal{P}(n)$ and if $f(X):=Q+$ $\sum_{j=1}^{m} A_{j}^{*} \cdot g(X) \cdot A_{j}$, then for each $X_{0} \in \mathcal{P}(n)$ we have $f^{n}\left(X_{0}\right) \rightarrow X^{*}$, as $n \rightarrow+\infty$.

Proof. It is easy to observe that $f(Q) \geq Q$. Next, as in [9] we obtain that there exists $a \in] 0,1\left[\right.$ such that $\|f(Y)-f(X)\|_{1} \leq a \cdot\|Y-X\|_{1}$ for each $X \leq Y$. Since 
$\mathcal{H}(n)_{\leq} \in I(g \times g)$ we immediately have $\mathcal{H}(n)_{\leq} \in I(f \times f)$. The conclusion follows from Theorem 4.3.

Next consider the equation

$$
X=Q-\sum_{j=1}^{m} A_{j}^{*} \cdot g(X) \cdot A_{j}
$$

Then we have:

Theorem 5.2. Let $Q \in \mathcal{P}(n)$ such that $Q>\sum_{j=1}^{m} A_{j}^{*} \cdot g(X) \cdot A_{j}$, for each $X \in \mathcal{H}(n)$ and let $g: \mathcal{P}(n) \rightarrow \mathcal{P}(n)$ be orbitally continuous. Suppose that:

(a) there exists $M>0$ such that:

(i) $\sum_{j=1}^{m} A_{j}^{*} A_{j}<M \cdot I_{n}$,

(ii) $\|\operatorname{tr}(g(Y)-g(X))\| \leq M^{-1} \cdot\|\operatorname{tr}(Y-X)\|$, for each $X \leq Y$.

(b) $\mathcal{H}(n) \leq \in I(g \times g)$.

Then equation (5.2) has a unique solution $X^{*}$ in $\mathcal{P}(n)$ and if $f(X):=Q-$ $\sum_{j=1}^{m} A_{j}^{*} \cdot g(X) \cdot A_{j}$, then for each $X_{0} \in \mathcal{P}(n)$ we have $f^{n}\left(X_{0}\right) \rightarrow X^{*}$, as $n \rightarrow+\infty$.

Proof. From the hypothesis it follows that $f(X)>0$ for each $X \in \mathcal{H}(n)$. Also $f(Q) \leq Q$. Moreover, $f$ satisfies the assertions $\|f(Y)-f(X)\|_{1} \leq a \cdot\|Y-X\|_{1}$ for each $X \leq Y$ and $\mathcal{H}(n) \in I(f \times f)$. The conclusion follows again by Theorem 4.3 .

Our final application is for a system of nonlinear equations in $\left(\mathbb{R}^{m}, \rightarrow, \leq\right)$.

Theorem 5.3. Consider the ordered L-space $\left(\mathbb{R}^{m}, \rightarrow, \leq\right)$ and $f: \mathbb{R}^{m} \rightarrow \mathbb{R}^{m}$. Suppose that:

(i) $\mathbb{R}_{\leq}^{m} \in I(f \times f)$,

(ii) $f$ is orbitally continuous,

(iii) there exists $x_{0} \in \mathbb{R}^{m}$ such that $\left(x_{0}, f\left(x_{0}\right)\right) \in \mathbb{R}_{\leq}^{m}$,

(iv) there exists a matrix $A \in M_{m m}\left(\mathbb{R}_{+}\right)$, such that:

(a) $A^{n} \rightarrow 0$, as $n \rightarrow \infty$,

$$
\left(\begin{array}{l}
\left\|f_{1}(x)-f_{1}(y)\right\| \\
\cdots \\
\left\|f_{m}(x)-f_{m}(y)\right\|
\end{array}\right) \leq A \cdot\left(\begin{array}{l}
\left\|x_{1}-y_{1}\right\| \\
\cdots \\
\left\|x_{m}-y_{m}\right\|
\end{array}\right),
$$

for each $x, y \in \mathbb{R}^{m}$, with $x \leq y$.

Then the equation $x=f(x)$ has a unique solution $x^{*} \in \mathbb{R}^{m}$ and $f^{n}\left(x_{0}\right) \rightarrow x^{*}$ as $n \rightarrow+\infty$, for all $x_{0} \in \mathbb{R}^{m}$.

\section{REFERENCES}

[1] T. A. Burton, Integral equations, implicit functions and fixed points, Proc. A.M.S., 124:23932390, 1996. MR1346965 (96j:45001)

[2] E. De Pascale, G. Marino and P. Pietromala, The use of the E-metric spaces in the search for fixed points, Le Mathematiche, 48:367-376, 1993. MR.1320676 (95m:54037)

[3] M. Fréchet, Les espaces abstraits, Gauthier-Villars, Paris, 1928. MR.1189135 (93g:01098)

[4] O. Hadžić, E. Pap and V. Radu, Generalized contraction mapping principles in probabilistic metric spaces, Acta Math. Hungar., 101:131-138, 2003. MR2011468 (2004i:54052)

[5] O. Hadžić and E. Pap, Fixed point theory in probabilistic metric spaces, Kluwer Acad. Publ., Dordrecht, 2001. MR1896451 (2003a:47113) 
[6] W. A. Kirk, B. Sims (editors), Handbook of metric fixed point theory, Kluwer Acad. Publ., Dordrecht, 2001. MR.1904271 (2003b:47002)

[7] J. Merryfield and J. D. Stein, A generalization of the Banach contraction principle, J. Math. Anal. Appl., 273:112-120, 2002. MR.1933019 (2003g:54100)

[8] A. Petruşel, Multivalued weakly Picard operators and applications, Scienticae Mathematicae Japonicae, 59:167-202, 2004. MR2027745 (2004j:47101)

[9] A. C. M. Ran and M.C. Reurings, A fixed point theorem in partially ordered sets and some applications to matrix equations, Proc. A.M.S., 132:1435-1443, 2004. MR2053350 (2005a:47112)

[10] I. A. Rus, Generalized contractions and applications, Cluj Univ. Press, 2001. MR 1947742 (2004f:54043)

[11] I. A. Rus, Picard operators and applications, Scientia Mathematicae Japonicae, 58:191-219, 2003. MR1987831 (2004m:47142)

[12] I. A. Rus, A. Petruşel, and G. Petruşel, Fixed point theory 1950-2000: Romanian contributions, House of the Book of Science, Cluj-Napoca, 2002. MR1947195 (2003h:47104)

[13] P. P. Zabreiko, K-metric and K-normed linear spaces: survey, Collect. Math., 48:825-859, 1997. MR.1602605 (99a:46010)

Department of Applied Mathematics, Babeş-Bolyai University Cluj-Napoca, KogăLNiCEAnu 1, 400084, Cluj-Napoca, Romania

E-mail address: petrusel@math.ubbcluj.ro

Department of Applied Mathematics, Babeş-Bolyai University Cluj-Napoca, KogăLNiceanu 1, 400084, Cluj-Napoca, Romania

E-mail address: iarus@math.ubbcluj.ro 